\title{
Human Papilloma Virus in cervical Cancer Carcinogenesis and Its Immunology
}

Maleeha Hussain

\section{Introduction}

Cervical cancer is one of the commonest cancers of women in developing world ${ }^{1}$. In developed countries well organized screening programme has successfully reduced the incidence. Cervical cancer is the first cancer that is cent percent attributable to a virus $^{2}$.

Molecular and epidemiological studies have conclusively established causal relationship between high risk human papilloma virus genotypes and cervical cancer. Papillomavirus are a very heterogeneous group of virus. They are widely distributed in nature but are highly species specific and not prone to mutation. Human papilloma virus is a non-enveloped double stranded DNA virus enclosed in a capsid shell. The 8 kilo base circular genome of HPV is made of early (E1 to E7) and 2 Late gene. The early genes are responsible for viral replication, transcription of non structural early proteins and assembly of new viral particles. The 2 late genes encode common capsid protein. The natural host immune response is directed to epitops on the $L 1$ protein. The $L 1$ protein when expressed via recombinant yeast or viral vectors folds and selfassembles into empty capsids or viral like particles. Antigenically and morphologically the virus like particle resemble wild virus and form the basis of current prophylactic vaccine. There is tropism of HPV infection for different tissues by various genotypes. Of them the genital types are $6.11,16$, 18 various $30 \mathrm{~s} 40 \mathrm{~s} 50 \mathrm{~s} 60 \mathrm{~s}$ and $70 \mathrm{~s}$. High risks are $16,18,31,33,38,39,45,51,52,56,58,59,68,73$ and 82 . Low risks are $6,11,40,42,43,44,54,61$, 70,72 and 81 . HPV 16 and 18 contribute to $70 \%$ of squamous cell carcinoma and 80 to $85 \%$ of adenocarcinoma of cervix. Human papilloma virus specifically infect epithelial cells of the skin or mucosa. They enter through minor abrasion of the squamous epithelium or through single cell junction of the squamocolumnar junction of the transformation zone of cervix and infect the basal cellular layer. In a proportionate of cases latency is maintained by a low copy number of viral genome in episomal form in the host nucleus. The complex life cycle of HPV is completed in the suprabasal compartment where the karatinocytes lose their ability to replicate and are terminally differentiated. As the epithelium is shed the full virions are ready to infect the next host. It is for this complex interaction the virus cannot be cultured in cell line and development of attenuated HPV vaccine is not possible. In high grade lesions and cervical cancer, HPV genome are covalently bonded or integrated into host chromosome. The E6 protein of high risk HPV binds with cancer suppressor gene p53, induces its degradation and removes control of host cell cycle. It also immortalizes cell by increasing telomerase activity. E7 gene product associates with the product of $\mathrm{Rb}$ gene, a cancer suppressor gene important in the negative control of cell growth. The E6 and E7 of low-risk HPV types weakly binds with p53 and Rb gene ${ }^{3,4}$.

With HPV infection there is no associated viremia, or inflammatory response. It is, as if the virus hides from immune system. The innate immune system which is nonspecific and not antigen specific is remsponsible for clearing some transient infections by the help of macrophage, monocytes and cytokines. In additions adaptive immune response occurs. The adaptive immune response to genital HPV is mainly cell mediated immunity. This is associated with lesion regression and possible inhibition of reinfection by same type of virus. There is also a humoral response in the form of type specific antibodies against capsid proteins $L 1$. These responses are slow to develop, weak and seen in only $60 \%$ of HPV-DNA positive in genital tract. Sero conversion occurs in approximately 8-12 months. The response is weak possibly because the viral capsid proteins are only expressed in the uppermost layer of HPV infected epithelium and are not efficiently presented to systemic immune system. The antibodies are neutralizing type and prevent the individual from further infection with that particular genotype. The antibody titre wanes with time and with this whether the natural protection persists or the individual becomes susceptible to reinfection is not clear. The virus can remain within basal epithelial cells at non-detectable levels, in latent state for many years and then become reactivated particularly 
with immune suppression or immune senescence 5 . Despite the low antibody level, seropositve individuals are protected against further viral challenge. This is the basis of vaccine development. Vaccines that generate neutralizing antibodies to HPV capsid protein will be effective prophylactically. Prophylactic HPV vaccines are based on major capsid protein L1 and L2. Recombinantly expressed $L 1$ the major capsid protein of HPV, self assembles to form empty viral capsids referred to virus like particles (VLP). Two HPV L1 VLP vaccines have been developed a quadrivalent vaccine (Gardasil) containing L1 VLP of 6/11/16/18 HPV types and a bivalent vaccine against $16 / 18 \mathrm{HPV}$. Since HPV vaccines are prophylactic vaccine the target population determination depends at which age individuals are first exposed to HPV infection. After vaccination peak titre is achieved 1 month after dose 3 after which detectable titres decline, until about month 18 when the rate of decline decreased considerably and titre appears to stabilize over next few months at or above titre observed in women with naturally acquired and cleared infection (ie. Those positive antibody and negative PCR-based assay for the same type of HPV). The aim of HPV vaccination is to prevent cervical cancer. All vaccinated individuals show rapid development of high titre of neutralizing IgG which are thought to transudate across cervical secretions into tissues and neutralize any wild type virus infection. Data correlating antibody persistence do not support booster dose. Even after reasonable coverage of vaccination programme cervical cancer screening should still continue as the target populations are only very young group. More over vaccine will not protect all HPV infection. In developing country where no cervical screening program exists, do vaccine can fight against cervical cancer is still a debate. It depend on the attitude of the women to screening and the extent, the country can afford the expensive vaccine $^{5,6,7}$.

\section{References}

1. World Health Organization. Control of cancer of the cervix uteri. Bull World Health Organ 1986; 64: 607-18.

2. Annual Report, 2005, Hospital cancer Registry, national Institute of Cancer Research \& Hospital.

3. Kim R Geisenger, Michael W Stanley, Stephen S. Raab, Jan F. Silverman, Andrea Abati. Modern Cytopathology, Churchill Livingstone 2004: pg 109-136.

4. William J Hoskins, Carlos A Perez, Robert C Young, Richard Barakat, Maurice Markman, Marius Randell. Principles \& Practice of Gynaecologic Oncology. Fourth edition. Lippincott Williams \& Wilkins

5. Naeal M Nour. Cervical cancer: A preventable death. Reviews in Obstetrics \& Gynaecology. 2009; Vol 2 No 4: 240-245.

6. Neerja Bhatla \& Elizabeth Joseph. Cervical cancer prevention \& role of human papilloma virus vaccines in India. Indian J Med res 130, September 2009, 334340.

7. Suzanee M Garland. Can cervical cancer be eradicated by prophylactic HPV vaccination? Challenges to vaccine implementation. Indian J Med Res 130, September 2009, pp 311-321. 